\title{
AMENDMENTS
}

\section{Publisher Correction: Controllable conductive readout in self-assembled, topologically confined ferroelectric domain walls}

Ji Ma, Jing Ma (1), Qinghua Zhang, Renci Peng, Jing Wang, Chen Liu, Meng Wang, Ning Li, Mingfeng Chen (1),

Xiaoxing Cheng, Peng Gao (D), Lin Gu, Long-Qing Chen, Pu Yu, Jinxing Zhang and Ce-Wen Nan

Correction to: Nature Nanotechnology https://doi.org/10.1038/s41565-018-0204-1, published online 23 July 2018.

In the version of this Article originally published, the corresponding author names in the author list appeared in the reverse order; they should have read 'Jinxing Zhang and Ce-Wan Nan'. The order of these authors' initials in the 'Correspondence and requests for materials' statement were similarly affected. These errors have now been corrected in all versions of the Article.

Published online: 31 July 2018

https://doi.org/10.1038/s41565-018-0247-3

\section{Author Correction: Plasmonic meta-electrodes allow intracellular recordings at network level on high-density CMOS-multi-electrode arrays}

Michele Dipalo (D), Giovanni Melle, Laura Lovato, Andrea Jacassi, Francesca Santoro, Valeria Caprettini, Andrea Schirato, Alessandro Alabastri (D), Denis Garoli, Giulia Bruno, Francesco Tantussi (iD and Francesco De Angelis

Correction to: Nature Nanotechnology https://doi.org/10.1038/s41565-018-0222-z, published online 13 August 2018.

In the version of this Article originally published, the affiliation for the author Francesca Santoro was incorrectly given; it should have been 'Center for Advanced Biomaterials for Healthcare, Istituto Italiano di Tecnologia, Napoli, Italy'. This has now been corrected in all versions of the Article. 